\title{
ESTIMATION OF COVID-19 INFECTION GROWTH RATE BASED ON THE IMBEDDING METHOD
}

\section{V. Derevich and A. A. Panova}

UDC 51-76

An analysis is presented of universal stages in the development of the COVID-19 virus infection epidemic. It is assumed that during a pandemic the growth rate in the number of infections in a country occurs similarly to the process of virion replication in an infected organism. The exponent of the growth rate of the number of infection cases reflects not only biomedical parameters of a virus infection but also distinctive features of the social behavior of the population of a country. The dynamics of the change in the growth rate exponents is simulated by a system of relaxation-type ordinary differential equations. In applied mathematics, based on the imbedding method, limit values are forecast for exponents of the growth rate of the number of cases using the currently available experimental data. These values "forecast ahead" are subsequently approached by the actual exponents of growth in the number of infectees. The search for unknown parameters is carried out by minimizing a specially constructed quadratic functional accounting for all confirmed cases of COVID-19 infection. The functional minimum is found via iterations by solving a system of ordinary differential equations.

Keywords: COVID-19 epidemic, number of infectees, quarantine, social behavior, imbedding method, growth rate in the number of infectees (infection cases).

Introduction. The coronavirus COVID-19 pandemic poses a substantial threat to modern civilization. The rapid spread of the epidemic has a destructive effect on the economy of many countries of the world. To develop efficient measures for localizing the epidemic and delivering medical care, it is necessary to have a forecast of the number of infection cases in a country for several days ahead. As shown by an analysis of the available experimental data, the dynamics of the epidemic development is determined not only by the biomedical parameters of the virus but also by the distinctive features of the social behavior of the population of the countries.

The proposed methods of simulating the COVID-19 epidemic can be divided into three classes. The first, the least sophisticated, class is based on the selection of an empirical function whose behavior is similar to the dynamics of change in the number of cases during an epidemic [1]. Use is made of the data on an epidemic that is already over, for example, in China. Efforts to transfer this methodology to forecasting a currently unfolding epidemic are inefficient, as a rule.

The second class may include models for describing the development of infectious disease epidemics proposed almost 100 years ago. They are SIR-type (Susceptible, Infected, Recovered/dead) models and their various modifications (for instance, $[2,3])$. In these popular models oriented toward long-term forecasts, use is made of a whole number of parameters and complicating closing relations that have to be adjusted for real data by the trial-and-error method on a daily basis for the COVID-19 epidemic [4]. The SIR approach is based on a mechanistic hypothesis of transferring infection among individuals. The main factor in SIR-type models is the reproduction rate, which shows the likely number of susceptible population members infected by one case. Efforts to estimate the reproduction rate for the COVID-19 pandemic in different countries lead to values differing by an order of magnitude [5].

An alternative to the mechanistic SIR model is the third class of investigations based on stochastic simulation of transferring not only of infection but also information in social interaction of individuals (social networks) [6-9]. The methods of forecasting the spread of infectious diseases created within interacting random social networks will probably be more efficient in the future than traditional SIR-type models. An overview of the literature on simulating the development of an infectious disease epidemic may include hundreds of references. We have only mentioned investigations reflecting fundamental areas of simulating the COVID-19 epidemic.

N. É. Bauman Moscow State Technical University, 5 2nd Baumanskaya Str., Building 1, Moscow, 105005, Russia; email: DerevichIgor@bmstu.ru,panova005@gmail.com. Translated from Inzhenerno-Fizicheskii Zhurnal, Vol. 94, No. 1, pp. 22-34, January-February, 2021. Original article submitted June 13, 2020. 
In a pandemic regime, the explosive pattern of the virus propagation and the specific features of the collective social behavior of individuals create a unified dynamic system whose laws of development are not quite clear at present. From a practical standpoint, it is express forecasts for a few days that are of value rather than long-term forecasts for several months, whose reliability is quite questionable. In [10], based on the Richard formula for the number of infectees, use is made of an ordinary trial and error search to establish the optimum values of the model's unknown parameters minimizing the root-mean square deviation of real and calculated values of the number of infectees at the initial stage of epidemic growth [11, 12]. At the stage of active epidemic development, this approach made it possible to forecast the number of cases in China for 15 days ahead. However, the use of this approach [10] for simulation of a full epidemic scenario is problematic.

By the time of publication of this paper, the coronavirus epidemic situation in the world would have changed radically. The manuscript was prepared when most of the countries of the world were at a stage of reduction in the virus damaging capacity due to the lockdown. However, at present, the premature lifting of the quarantine restrictions has resulted in the development of a second COVID-19 epidemic wave. Therefore, the results of this investigation should be considered as a reconstruction of the scenario of the first COVID-19 epidemic wave.

We can distinguish three universal stages in the COVID-19 virus pandemic that are observed in many countries of the world. Identifying these stages on the basis of analyzing only curves for infected population growth is unpromising, since these curves are similar for all epidemic-affected countries. There is significantly more information in a curve for a daily growth in the number of cases. An analysis of the epidemic development indicates that the growth in the number of infectees is satisfactorily approximated by an exponential dependence with a time-variable exponent. As shown further in the paper, in the time interval of operation of each stage there is, in practice, a constant limiting exponent that is subsequently approached by the actual value of the exponent of the growth rate in the number of cases. This limiting exponent is predicted within quite a short initial interval of time that is substantially shorter than the duration of the stage. The forecast of the limiting value of the exponent is a "prediction ahead," since it is to this value that the actual exponent of the growth in the number of cases drops with the passage of time.

In this investigation, we only use the number of confirmed cases of infection whose changing dynamics is adequately reflective of not only the virus reproductive capacity but also of the specific features of the population social behavior. The proposed methods of analysis of the pandemic development make it possible, based on the data on the number of infectees alone, to monitor the specific features of the stages and to ensure satisfactory accuracy in predicting the number of cases for fifteen-thirty days ahead. It is obvious that the forecast accuracy is determined not only by the mathematical model but also by the reliability of the presented experimental data. We use the results of registration of the number of cases from the online site at COVID-19 Dashboard by the Center for Systems Science and Engineering (CSSE) at Johns Hopkins University (JHU).

Due to the limited volume of the paper, we select a number of countries to illustrate the ideas behind the investigation. It is Russia where there is a clear manifestation of the national distinctions of the quarantine resulting in a substantial rise in the number of cases and a delay in approaching the stage of reduction in the virus damaging capacity. It is Italy that was able to get out of an initially unfavorable situation to a stage of stabilizing the number of cases due to a rather strict observation of quarantine restrictions. It is Sweden where there is no strict quarantine, which results in a substantial increase in the epidemic duration compared to such countries of Europe as Germany, Italy, Spain, and France.

Basic Equations. We assume that during a pandemic the biological mechanism of virus replication and the specific features of the social behavior of a country's population form a single system in which the rise in the number of cases occurs similarly to the growth of pathogenic virions in the infected organism of one individual. Note that the number of cases in all the countries is still below $2 \%$ of the countries' population. Therefore, we write the equation for the number of cases $N(t)$ in the form

$$
\frac{d}{d t} N(t)=\beta(t) N(t), \quad N\left(t_{0}\right)=N_{0} .
$$

Here, $N_{0}$ is the number of infectees at the initial instant $t_{0}$. The actual number of cases follows from Eq. (1):

$$
N(t)=N_{0} \exp \left\{\int_{t_{0}}^{t} \beta(\xi) d \xi\right\} .
$$

Next, the model is constructed on the basis of a description of the dynamics of change in the exponent $\beta(t)$ in Eq. (1). We distinguish three universal stages through which an overwhelming majority of countries pass. At the first stage, the epidem- 
ic development begins with the migration of infected individuals into a country with a subsequent uncontrolled epidemic development. Then, at the quarantine stage, administrative measures of restrictive nature come into force. We consider that the biological parameters of the COVID-19 virus strain are identical in all the countries. Therefore, the infected population growth rate fully depends on the geographical features of a country and the collective behavior of its population in implementing quarantine measures. The next stage in the degeneration of the virus infection is associated with a decline in the virus damaging capacity and a reduced likelihood of virus transmission between individuals during the quarantine regime. The transition between different epidemic stages does not occur instantaneously. There is a period of adaptation to new conditions that can take several days. Therefore, we simulate the dynamics of change of the exponent in the growth rate with a system of relaxation-type equations. At the first stage, the change in the exponent $\beta(t)$ of the growth rate is described by the equation

$$
\frac{d \beta}{d t}=\frac{1}{T^{*}}\left(\beta^{*}-\beta\right),\left.\quad \beta\right|_{t=t_{0}}=\beta_{0}, \quad t_{0} \leq t<t^{*} .
$$

Here, $\beta_{0}$ is the initial value related to the migration of infectees to the country; $T^{*}$ is the characteristic time scale of transition to a stage of uncontrolled epidemic development; $\beta^{*}$ is the limiting value of the rate exponent at the stage of uncontrolled growth; $t^{*}$ is the characteristic time of the beginning of the quarantine regime.

In Eq. (2), the unknown parameters are the characteristic time of relaxation $T^{*}$, the initial growth rate exponent $\beta_{0}$, and the limiting value of the exponent $\beta^{*}$. These values reflect specific features of social behavior and are determined by the experimental data of the first days after the beginning of the stage and remain, in practice, constant for the duration of the entire period. This will be illustrated in the "Calculation Results" section. Also, note that due to the unreliability of the diagnostics of the initial number of cases, the parameter $N_{0}$ requires correction, too.

The second stage is quarantine. After the stage of uncontrolled growth, the exponent relaxes to the new limiting value $\beta^{* *}$, whose magnitude depends substantially on the national distinctions in observing quarantine restrictions:

$$
\frac{d \beta}{d t}=\frac{1}{T^{* *}}\left(\beta^{* *}-\beta\right),\left.\quad \beta\right|_{t=t^{*}}=\beta\left(t^{*}\right), \quad t^{*} \leq t<t^{* *} .
$$

Here, $T^{* *}$ is the characteristic time of relaxing adaptation to quarantine conditions; $\beta\left(t^{*}\right)$ is the value of the growth rate exponent at the instant of time of the quarantine introduction; $t^{* *}$ is the characteristic time of the stage of decline in the virus damaging capacity.

In Eq. (3), the unknown parameters whose values are estimated by the data of the first quarantine days are $T^{* *}$ and $\beta^{* *}$. The characteristic time of the beginning of the stage of decline in the virus damaging capacity $t^{* *}$ is determined empirically by registering a reduction in the daily increment of the number of cases and turns out to be close for a number of European countries and the USA, which is indicative of the similarity of biological parameters of the COVID-19 virus strain in different regions. At the stage of stabilization in the number of cases, the equation for the growth rate indicator has the form

$$
\frac{d \beta}{d t}=\frac{1}{T^{* * *}}\left(\beta^{* * *}-\beta\right),\left.\quad \beta\right|_{t=t^{* *}}=\beta\left(t^{* *}\right), \quad t \geq t^{* *},
$$

where $T^{* * *}$ is the characteristic time of relaxation to the limiting value $\beta^{* * *} \rightarrow 0 ; \beta\left(t^{* *}\right)$ is the value of the exponent at the instant of time of the beginning of the stage of epidemic degradation. The system of equations (1)-(4) has an analytical solution for both the exponent and the logarithm of the number of cases:

$$
\beta(t)=\left\{\begin{array}{l}
\beta_{0} \exp \left(-\frac{t-t_{0}}{T^{*}}\right)+\beta^{*}\left[1-\exp \left(-\frac{t-t_{0}}{T^{*}}\right)\right], \quad t_{0} \leq t<t^{*}, \\
\beta\left(t^{*}\right) \exp \left(-\frac{t-t^{*}}{T^{* *}}\right)+\beta^{* *}\left[1-\exp \left(-\frac{t-t^{*}}{T^{* *}}\right)\right], \quad t^{*} \leq t<t^{* *}, \\
\beta\left(t^{* *}\right) \exp \left(-\frac{t-t^{* *}}{T^{* * *}}\right)+\beta^{* * *}\left[1-\exp \left(-\frac{t-t^{* *}}{T^{* * *}}\right)\right], \quad t>t^{* *} ;
\end{array}\right.
$$




$$
\ln N(t)=\left\{\begin{array}{l}
\ln N_{0}+\beta^{*}\left(t-t_{0}\right)+\left(\beta_{0}-\beta^{*}\right) T^{*}\left[1-\exp \left(-\frac{t-t_{0}}{T^{*}}\right)\right], \quad t_{0} \leq t<t^{*}, \\
\ln N\left(t^{*}\right)+\beta^{* *}\left(t-t^{*}\right)+\left[\beta\left(t^{*}\right)-\beta^{* *}\right] T^{* *}\left[1-\exp \left(-\frac{t-t^{*}}{T^{* *}}\right)\right], \quad t^{*} \leq t<t^{* *}, \\
\ln N\left(t^{* *}\right)+\beta^{* * *}\left(t-t^{* *}\right)+\left[\beta\left(t^{* *}\right)-\beta^{* * *}\right] T^{* * *}\left[1-\exp \left(-\frac{t-t^{* *}}{T^{* * *}}\right)\right], \quad t \geq t^{* *} .
\end{array}\right.
$$

The values of the number of cases and of the exponent in the growth rate at the instants of time of the change of stages are equal to

$$
\begin{gathered}
\ln N\left(t^{*}\right)=\ln N_{0}+\beta^{*}\left(t^{*}-t_{0}\right)+\left(\beta_{0}-\beta^{*}\right) T^{*}\left[1-\exp \left(-\frac{t^{*}-t_{0}}{T^{*}}\right)\right], \\
\ln N\left(t^{* *}\right)=\ln N\left(t^{*}\right)+\beta^{* *}\left(t^{* *}-t^{*}\right)+\left[\beta\left(t^{*}\right)-\beta^{* *}\right] T^{* *}\left[1-\exp \left(-\frac{t^{* *}-t^{*}}{T^{* *}}\right)\right], \\
\beta\left(t^{*}\right)=\beta_{0} \exp \left(-\frac{t^{*}-t_{0}}{T^{*}}\right)+\beta^{*}\left[1-\exp \left(-\frac{t^{*}-t_{0}}{T^{*}}\right)\right], \\
\beta\left(t^{* *}\right)=\beta\left(t^{*}\right) \exp \left(-\frac{t^{* *}-t^{*}}{T^{* *}}\right)+\beta^{* *}\left[1-\exp \left(-\frac{t^{* *}-t^{*}}{T^{* *}}\right)\right] .
\end{gathered}
$$

Thus, we have a selection of unknown parameters $\beta_{0}, \beta^{*}, \beta^{* *}, \beta^{* * *}, T^{*}, T^{* *}, T^{* * *}$, and $\ln N_{0}$ which determine the growth dynamics of the number of cases at all epidemic stages. It turns out that these parameters calculated for a short time interval using the experimental data vary insignificantly during the first epidemic wave. In fact, we determine parameters in the future, which the actual values of the growth rate exponent for the number of cases subsequently approach. In our view, the possibility of forecasting the model parameters into the future is due to the strict biological laws of virus replication and the conservative tradition of the national behavior of the countries' population.

The forecast of unknown parameters "ahead in time" is similar to the imbedding method in applied mathematics [13]. The idea of the imbedding method is the introduction of additional parameters closing the problem. The specific values of these parameters are estimated on the basis of minimizing a specially constructed functional. Note that the methods of variation analysis are actively used in solving inverse problems of various technical applications (for example, [14, 15]).

Minimized Functional. We define a quadratic functional whose minimum ensures the optimum values of the unknown parameters $\beta_{0}, \beta^{*}, \beta^{* *}, \beta^{* * *}, T^{*}, T^{* *}, T^{* * *}$, and $\ln N_{0}$ as

$$
\Phi\left(\beta_{0}, \beta^{*}, \beta^{* *}, \beta^{* * *}, T^{*}, T^{* *}, T^{* * *}, \ln N_{0}\right)=\sum_{k=1}^{K}\left[\ln N\left(t^{(k)}\right)-\ln N_{\text {inf }}^{(k)}\right]^{2} .
$$

Here, $K$ is the number of instants of time since the epidemic beginning for which, by the instant of time $t^{(K)}$, there are experimental data, $k=1, \ldots, K$.

The process of functional minimization is iterative. We search for the minimum relying on a mechanical analogy. We consider that a quadratic functional is a force-field potential in which a material point is moving. The velocity of the point's movement to the minimum is proportional to the potential antigradient. On the strength of this analogy, we write a system of ordinary differential equations for an iterative search of unknown parameters:

$$
\begin{aligned}
& \frac{d \beta_{0}}{d s}=-\frac{\partial}{\partial \beta_{0}} \Phi\left(\beta_{0}, \beta^{*}, \beta^{* *}, \beta^{* * *}, T^{*}, T^{* *}, T^{* * *}, \ln N_{0}\right), \\
& \frac{d \beta^{*}}{d s}=-\frac{\partial}{\partial \beta^{*}} \Phi\left(\beta_{0}, \beta^{*}, \beta^{* *}, \beta^{* * *}, T^{*}, T^{* *}, T^{* * *}, \ln N_{0}\right), \\
& \frac{d \beta^{* *}}{d s}=-\frac{\partial}{\partial \beta^{* *}} \Phi\left(\beta_{0}, \beta^{*}, \beta^{* *}, \beta^{* * *}, T^{*}, T^{* *}, T^{* * *}, \ln N_{0}\right),
\end{aligned}
$$




$$
\begin{aligned}
& \frac{d \beta^{* * *}}{d s}=-\frac{\partial}{\partial \beta^{* * *}} \Phi\left(\beta_{0}, \beta^{*}, \beta^{* *}, \beta^{* * *}, T^{*}, T^{* *}, T^{* * *}, \ln N_{0}\right), \\
& \frac{d T^{*}}{d s}=-\frac{\partial}{\partial T^{*}} \Phi\left(\beta_{0}, \beta^{*}, \beta^{* *}, \beta^{* * *}, T^{*}, T^{* *}, T^{* * *}, \ln N_{0}\right), \\
& \frac{d T^{* *}}{d s}=-\frac{\partial}{\partial T^{* *}} \Phi\left(\beta_{0}, \beta^{*}, \beta^{* *}, \beta^{* * *}, T^{*}, T^{* *}, T^{* * *}, \ln N_{0}\right), \\
& \frac{d T^{* * *}}{d s}=-\frac{\partial}{\partial T^{* * *}} \Phi\left(\beta_{0}, \beta^{*}, \beta^{* *}, \beta^{* * *}, T^{*}, T^{* *}, T^{* * *}, \ln N_{0}\right), \\
& \frac{d}{d s} \ln N_{0}=-\frac{\partial}{\partial \ln N_{0}} \Phi\left(\beta_{0}, \beta^{*}, \beta^{* *}, \beta^{* * *}, T^{*}, T^{* *}, T^{* * *}, \ln N_{0}\right) .
\end{aligned}
$$

The complete system of equations is presented in the Appendix.

A similar method of searching for the minimum was implemented in [16] for calculating thermodynamic equilibrium of a multicomponent two-phase system. The system (8) is integrated until it reaches the steady-state values of the sought parameters and the potential minimum. The computation program was implemented in the Mathcad language using the Radau method for numerical integration of a system of stiff ordinary differential equations.

Calculation Results. Figures 1 and 2 show calculation results based on experimental results of June 12, 2020. Figure 1 shows the results of calculating the time variation of the logarithm of the number of COVID-19 cases for Russia and Italy. It is seen that the instants of time for the beginning of the quarantine introduction $t^{*}$ and those for the beginning of the virus degradation $t^{* *}$ are separated by regions with a varied pattern of infected population growth. It can also be pointed out that by the time of the quarantine introduction, the epidemiological situation in Russia was significantly better than in Italy. However, due to the national peculiarities, the number of cases in Russia rose more than 20 times during the quarantine compared to the number of COVID-19 cases before the quarantine introduction. The observance of quarantine conditions in Italy led to the expected positive effect, viz. an apparent effort to achieve a state of stabilization in the number of cases. As shown in Fig. 1, it was going to take Russia quite some time to reach the "plateau."

The universal epidemic stages for Russia, Italy, and Sweden are illustrated in Fig. 2, which presents the time variation of the exponent of the epidemic growth rate. We can see that there are three stages. At the first stage, the maximum infection rate is observed at the initial instant of time. This is due to the migration of infectees to the country. With the passage of time, a value that is characteristic of uncontrolled epidemic growth settles for the growth rate exponent. In this case, the values of the exponent $\beta^{*}$ at the stage of uncontrolled epidemic development in Russia and Italy are close. However, at the quarantine stage, the values of the exponent $\beta^{* *}$ in Italy is substantially lower than in Russia. At the third stage of decline in the virus damaging capacity, the exponents for Russia and Italy monotonically decrease; however, the value of the exponent in Russia is significantly higher than in Italy. Despite the absence of a strict quarantine, the population of Sweden is adapting quite quickly to the new social epidemic conditions. We can see that the epidemic growth rate exponent at the stage of decline in the virus damaging capacity is substantially higher in Sweden than in Italy.

Figures 3-5 show the results of reconstruction and verification of the forecasting ability of the model for Russia (Fig. 3), Italy (Fig. 4), and Sweden (Fig. 5). To illustrate the forecasting properties of the model, we calculated the parameters using the experimental data from Region I. These data were used to calculate the epidemic parameters in Region II and to compare them with experimental values. We present the processing of data until the instant of time of the beginning of the second COVID-19 epidemic wave.

Figures 3-5 show the qualitative difference in the dynamics of the daily increment in the number of cases. Whereas in Italy, after the time of the population's adaptation to the quarantine conditions, there is a monotonic decrease in the increment of growth in the number of cases, in Russia, there is a rise in the increment of growth of the number of infectees until the instant of time of decline in the virus damaging capacity. There is a fundamental difference in the pattern of daily incremental growth in the number of cases in Sweden where a strict quarantine has not been introduced. Here, due to the organized social behavior of the country's population, the daily incremental growth in the number of cases varies in the vicinity of a certain value. However, at the expected stage of decline in the virus activity, we do not observe a tendency to reduction in the daily number of infections in Sweden. Note that, with correction for the time of the initial registration of the first COVID-19 infections, the decline in the virus activity in different countries occurred approximately at one and the same time.

The accuracy of approximation of the number of cases in the model is illustrated by a relative error: 

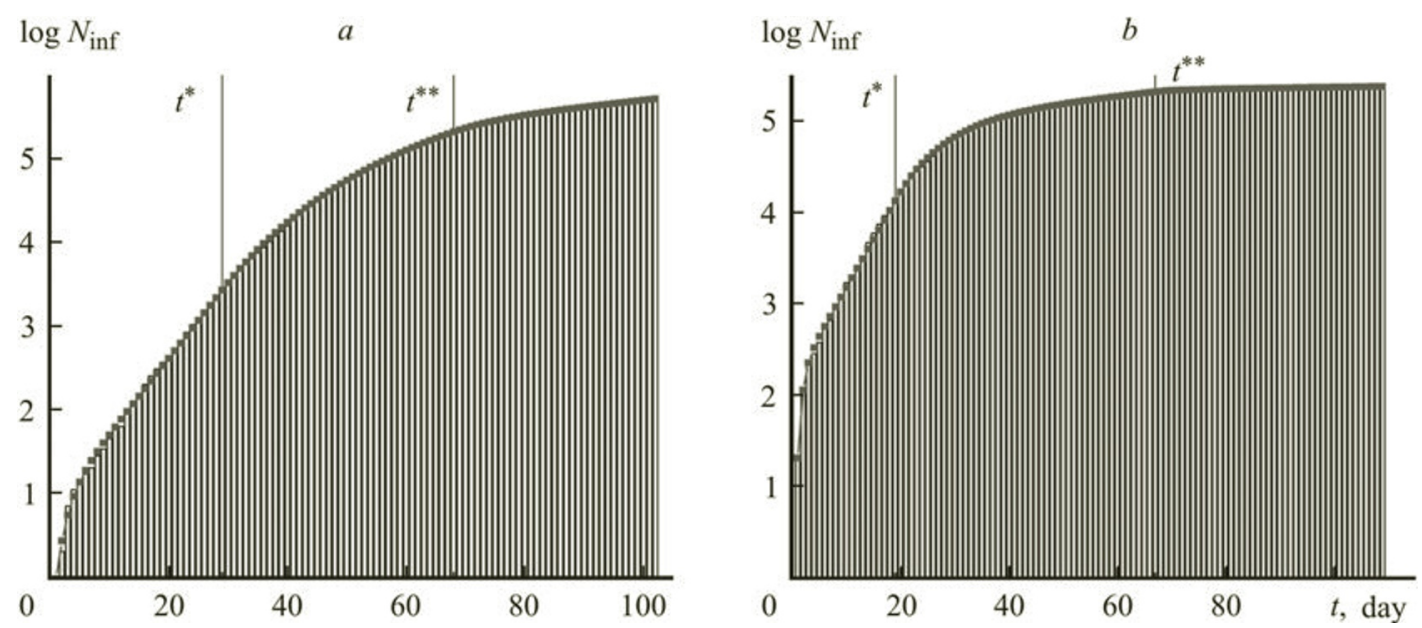

Fig. 1. Logarithms of the numbers of cases in Russia (a) and Italy (b): the columns are the experimental data; the dots are the results of calculation by the model.
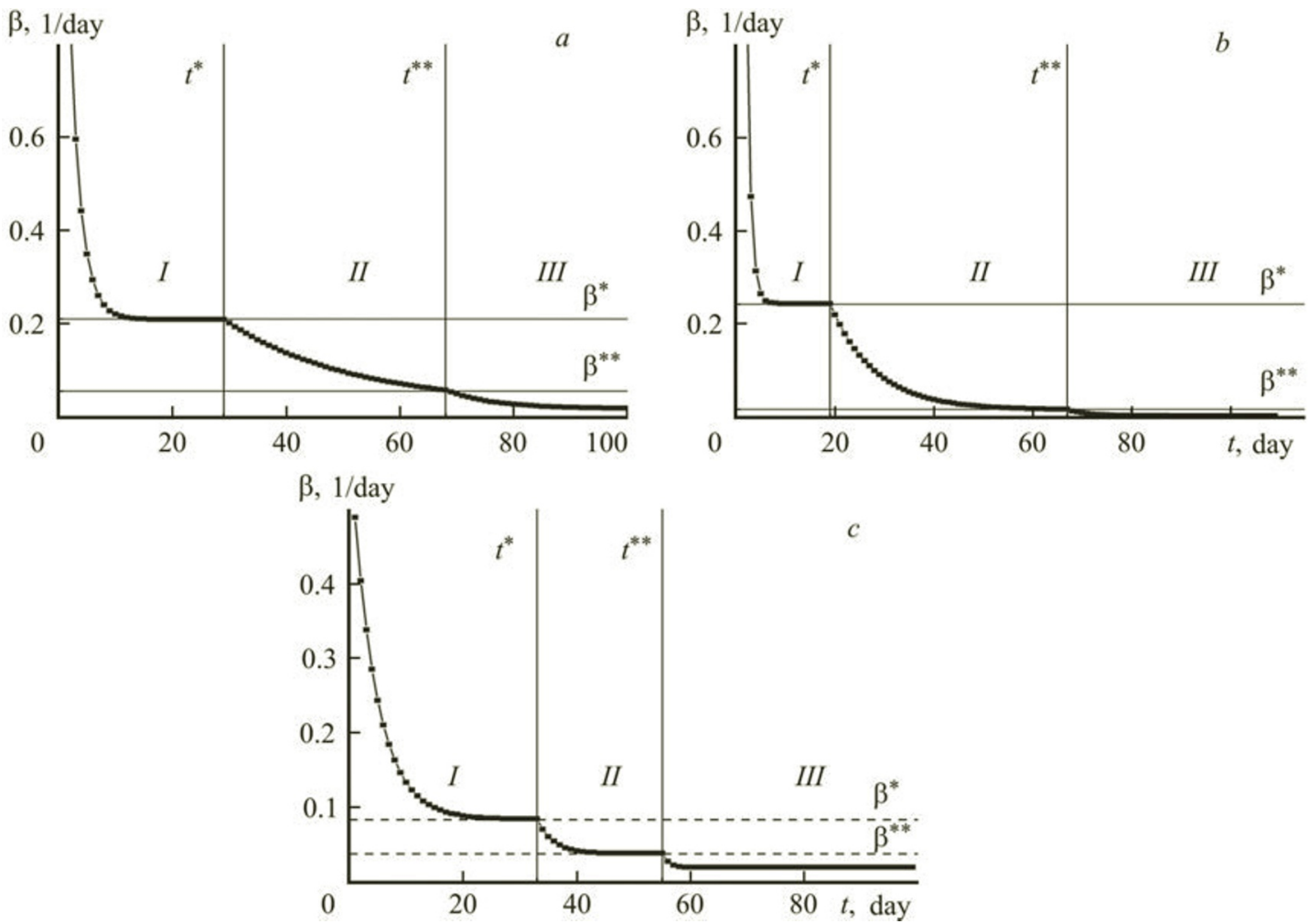

Fig. 2. Exponents for the infected population growth rate in Russia (a), Italy (b), and Sweden (c). Stages: I) migration of infected persons to the country and uncontrolled epidemic development; II) quarantine; III) decline in the virus damaging capacity.

$$
\varepsilon=\frac{N_{\text {inf }}^{(k)}-N\left(t^{(k)}\right)}{N_{\text {inf }}^{(k)}} .
$$

The maximum error at the first stage in all three countries is probably caused by the imperfection of the initial infection registration mechanism. At the subsequent epidemic stages, the approximation error becomes stable and does not exceed 5\%. Italy and many countries of Europe where a strict quarantine was observed reached a COVID-19 epidemic stage related to 

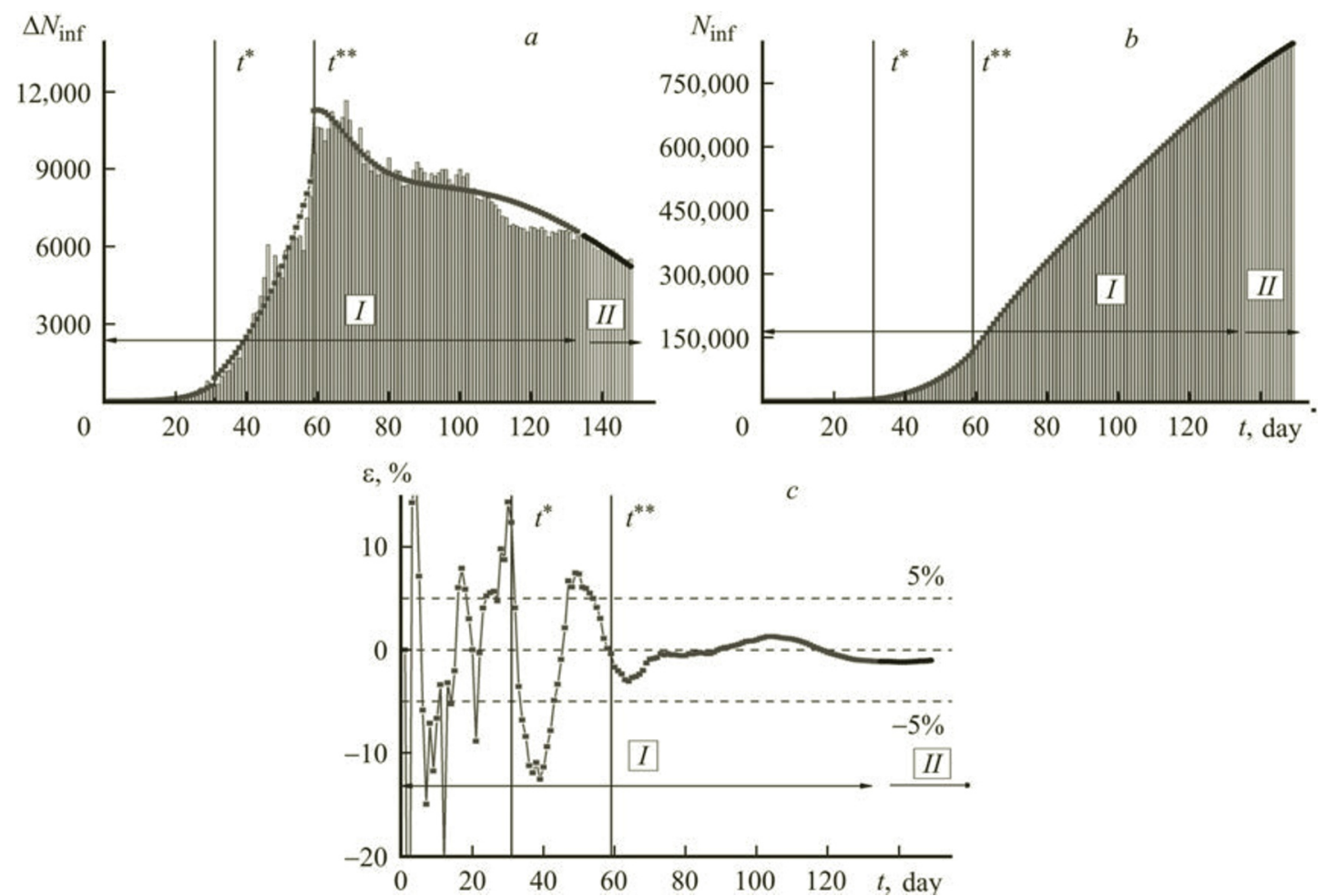

Fig. 3. Daily increments in the number of infections (a), total number of infectees (b), and error in approximating the number of cases (c) in Russia. The histograms are the experimental data, the dots are the calculation by the model; I) real data region (July 30, 2020); II) forecast region.
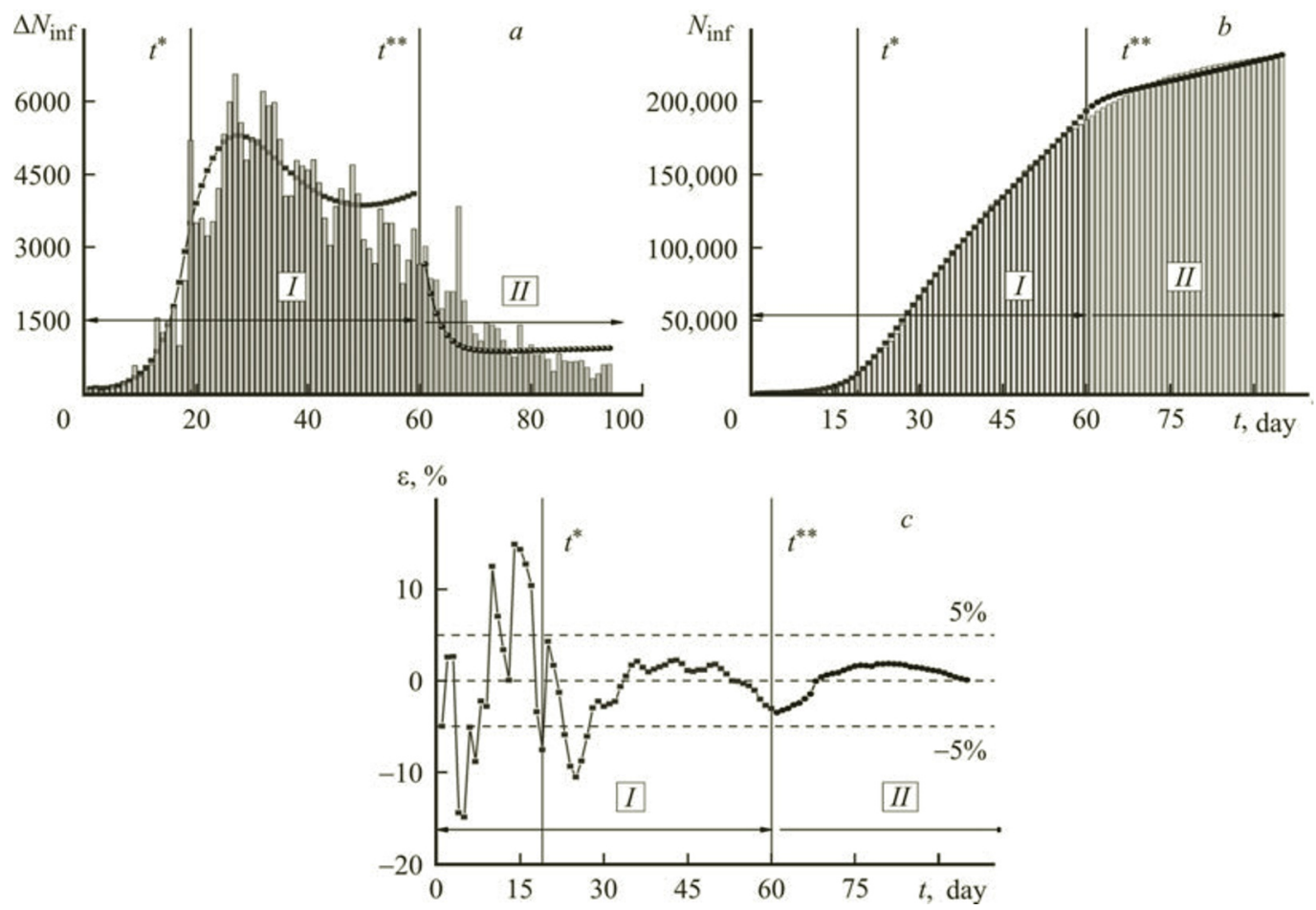

Fig. 4. Daily increments in the number of infections (a), total number of infectees (b), and error in approximating the number of cases (c) in Italy: I) real data region (May 28, 2020); II) forecast region. 


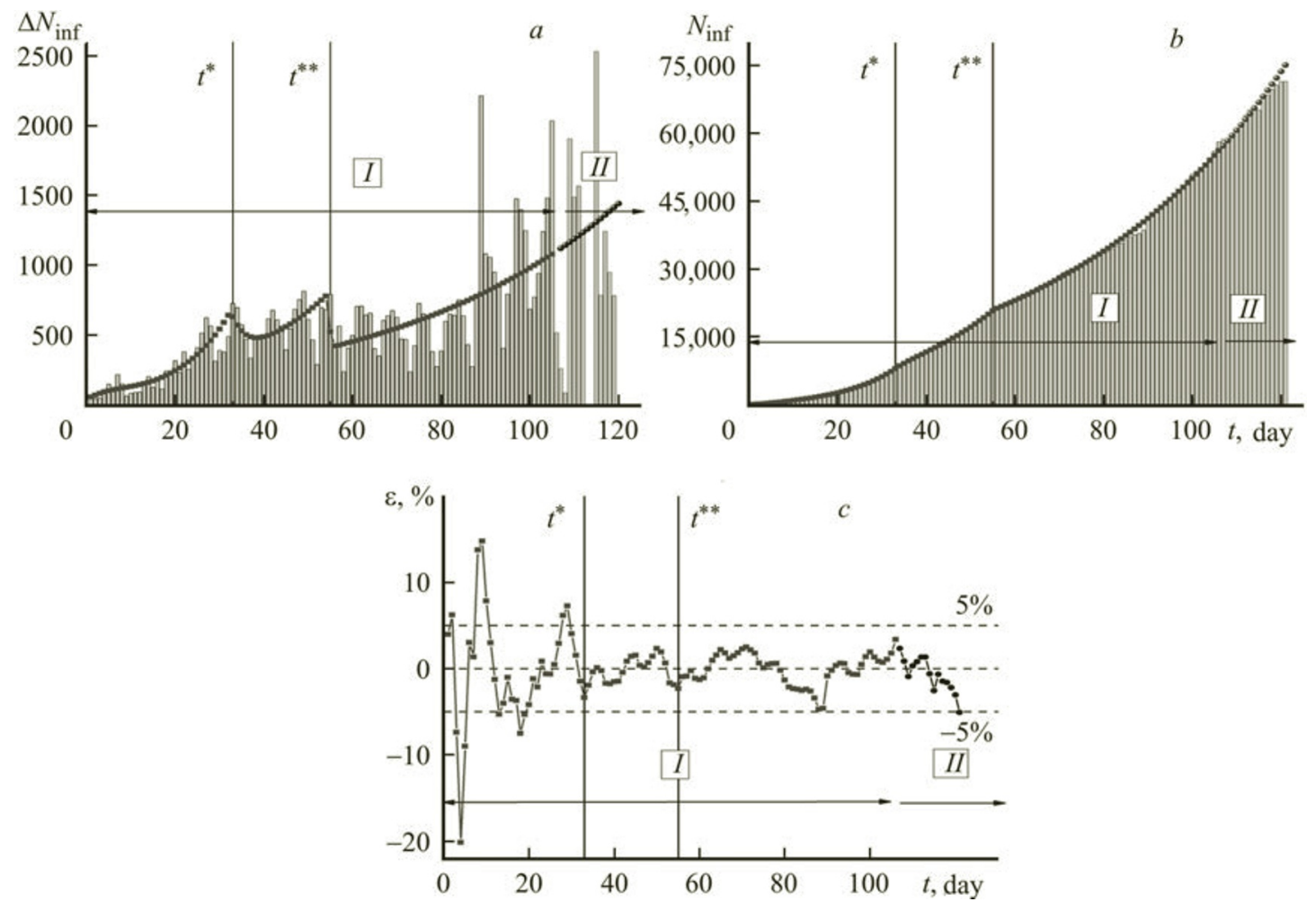

Fig. 5. Daily increments in the number of infections (a), total number of infectees (b), and error in approximating the number of cases (c) in Sweden: I) real data region (July 4, 2020); II) forecast region.

a decline in the virus damaging capacity. However, the premature lifting of the lockdown regime in the countries during the summer vacation period resulted in the development of the intensely propagating second COVID-19 epidemic wave. In this paper, we have presented an analysis of only the first coronavirus epidemic wave.

Conclusions. We propose a model for approximating the time dependence of the growth rate in the number of infections. To estimate the model parameters, use is made of the imbedding method of applied mathematics and of the experimental data on the number of COVID-19-cases.

Using the example of Russia, Italy, and Sweden, we present an analysis of the universal stages of the first COVID-19 epidemic wave observed in many countries of the world. Countries have been selected with a fundamentally different pattern of social behavior. It has been shown that the national distinctions of observing a quarantine have a substantial impact on the epidemic spread dynamics. For example, in Italy, at the stage of uncontrolled epidemic development, the number of cases was much higher than in Russia. The observance of strict quarantine measures in Italy has resulted in a substantial reduction in the epidemic propagation rate compared to Russia. In Sweden, where a strict quarantine regime has not been introduced, we can expect a substantially longer stage of COVID-19 epidemic degradation than for other European countries.

Acknowledgment. The investigation was supported by a grant from the Russian Foundation for Basic Research (Project No. 20-08-01061).

\section{NOTATION}

$k$ and $K$, serial number of the instant of time and the number of registered cases of infection by the instant of time $t^{(K)} ; N$, number of cases calculated by the model; $N_{\text {inf }}^{(k)}$, real number of cases at the instant of time $t^{(K)} ; s$, iteration parameter; $T$, characteristic time of relaxation, day; $t$, current time day; $\beta$, exponent for the growth rate in the number of cases, $1 /$ day; $\varepsilon$, relative error in approximating the number of cases; $\Theta(x)$, step function; $\Phi$ and $\varphi$, minimized functional and functional component. Indices: 0 , initial values; ${ }^{*}, *$, and ${ }^{* * *}$, parameters at the stage of uncontrolled growth, quarantine, and decline in the virus damaging capacity. 


\section{REFERENCES}

1. X. Fu, Q. Ying, T. Zeng, T. Long, and Y. Wang, Simulating and forecasting the cumulative confirmed cases of SARSCoV-2 in China by Boltzmann function-based regression analyses, J. Infection, Lett. Ed., 80, Issue 5, 602-605 (2020).

2. W. O. Kermack and A. G. McKendrick, A contribution to the mathematical theory of epidemics, Proc. Roy. Soc. A: Math., Phys. Eng. Sci., 115, No. 772, 700-721 (1927).

3. Z. Wanga, C. T. Bauch, S. Bhattacharyya, et al., Statistical physics of vaccination, Phys. Rep., 664, 1-113 (2016).

4. S. Flaxman, S. Mishra, A. Gandy, et al., Estimating the number of infections and the impact of nonpharmaceutical interventions on COVID-19 in 11 European countries, Imperial College London (30-03-2020); https://doi. org/10.25561/77731.

5. N. M. Ferguson, D. Laydon, G. Nedjati-Gilani, et al., Impact of non-pharmaceutical interventions (NPIs) to reduce COVID-19 mortality and healthcare demand, Imperial College London (16-03-2020); https://doi.org/10.25561/77482.

6. S. Eubank, I. Eckstrand, B. Lewis, et al., Commentary on Ferguson et al. impact of non-pharmaceutical interventions (NPIs) to reduce COVID-19 mortality and healthcare demand, Bull. Math. Biol., 82, No. 52 (2020); https://doi. org/10.1007/s11538-020-00726-X.

7. L. Lü, D. Chen, X.-L. Ren, Q.-M. Zhang, Y.-C. Zhang, and T. Zhou, Vital nodes identification in complex networks, Phys. Rep., 650, 1-63 (2016).

8. J. Enrighta and R. R. Kao, Epidemics on dynamic networks, Epidemics, 24, 88-97 (2018).

9. T. I. Vasylyeva, S. R. Friedman, D. Paraskevis, and G. Magiorkinis, Integrating molecular epidemiology and social network analysis to study infectious diseases: Towards a socio-molecular era for public health, Infect., Genet. Evol., 46, 248-255 (2016).

10. K. Roosa, Y. Lee, R. Luo, A. Kirpich, R. Rothenberg, J. M. Hyman, P. Yan, and G. Chowell, Real-time forecasts of the COVID-19 epidemic in China from February 5th to February 24th, 2020, Infect. Dis. Model., 5, 256-263 (2020).

11. X.-S. Wang, J. Wu, and Y. Yang, Richards model revisited: Validation by and application to infection dynamics, J. Theor. Biol., 313, 12-19 (2012).

12. G. Chowell, L. Sattenspiel, S. Bansal, and C. Viboud, Mathematical models to characterize early epidemic growth: A review, Phys. Life Rev., 18, 66-97 (2016).

13. J. Casti and R. Kalaba, Imbedding Methods in Applied Mathematics [Russian translation], Mir, Moscow (1976).

14. L. Yu. Levin, M. A. Semin, and A. V. Zaitsev, Solution of an inverse Stefan problem in analyzing the freezing of groundwater in a rock mass, J. Eng. Phys. Thermophys., 91, No. 3, 611-618 (2018).

15. A. G. Vikulov and A. V. Nenarokomov, Identification of mathematical models of the heat excange in space vehicles, $J$. Eng. Phys. Thermophys., 92, No. 1, 29-42 (2019).

16. I. V. Derevich and A. A. Panova, Calculation of thermodynamic equilibrium of a multicomponent two-phase system based on minimization of the Gibbs potential, J. Eng. Phys. Thermophys., 93, No. 2, 247-260 (2020).

\section{APPENDIX}

We write the minimized functional (7) in the form

$$
\begin{aligned}
& \Phi\left(\beta_{0}, \beta^{*}, \beta^{* *}, \beta^{* * *}, T^{*}, T^{* *}, T^{* * *}, \ln N_{0}\right)=\sum_{k=1}^{K}\left\{\left[\varphi^{*}\left(k<k^{*}\right)\right]^{2} \Theta\left(k^{*}-1-k\right)\right. \\
+ & {\left.\left[\varphi^{* *}\left(k^{*} \leq k<k^{* *}\right)\right]^{2} \Theta\left(k-k^{*}\right) \Theta\left(k^{* *}-1-k\right)+\left[\varphi^{* * *}\left(k^{* *} \leq k\right)\right]^{2} \Theta\left(k-k^{* *}\right)\right\} . }
\end{aligned}
$$

Here $k^{*}$ and $k^{* *}$ are serial numbers of the days corresponding to the instants of time $t^{*}$ and $t^{* *}$, and the step function $\Theta(x)$ is equal to

$$
\Theta(x)= \begin{cases}0, & x<0 \\ 1, & x \geq 0\end{cases}
$$

The functional components have the following form:

$$
\varphi^{*}\left(k<k^{*}\right)=\ln N_{0}+\beta^{*}\left(t^{(k)}-t_{0}\right)+\left(\beta_{0}-\beta^{*}\right) T^{*}\left[1-\exp \left(-\frac{t^{(k)}-t_{0}}{T^{*}}\right)\right]-\ln N_{\text {inf }}^{(k)},
$$




$$
\begin{aligned}
& \varphi^{* *}\left(k^{*} \leq k<k^{* *}\right)=\ln N\left(t^{*}\right)+\beta^{* *}\left(t^{(k)}-t^{*}\right)+\left[\beta\left(t^{*}\right)-\beta^{* *}\right] T^{* *}\left[1-\exp \left(-\frac{t^{(k)}-t^{*}}{T^{* *}}\right)\right]-\ln N_{\text {inf }}^{(k)}, \\
& \varphi^{* * *}\left(k^{* *} \leq k\right)=\ln N\left(t^{* *}\right)+\beta^{* * *}\left(t^{(k)}-t^{* *}\right)+\left[\beta\left(t^{* *}\right)-\beta^{* * *}\right] T^{* * *}\left[1-\exp \left(-\frac{t^{(k)}-t^{* *}}{T^{* * *}}\right)\right]-\ln N_{\text {inf }}^{(k)} .
\end{aligned}
$$

The derivatives of these components with respect to sought parameters are written in the following way:

derivative with respect to $\beta_{0}$

$$
\begin{gathered}
\frac{\partial}{\partial \beta_{0}} \varphi^{*}\left(k<k^{*}\right)=T^{*}\left[1-\exp \left(-\frac{t^{(k)}-t_{0}}{T^{*}}\right)\right], \\
\frac{\partial}{\partial \beta_{0}} \varphi^{* *}\left(k^{*} \leq k<k^{* *}\right)=T^{*}\left[1-\exp \left(-\frac{t^{*}-t_{0}}{T^{*}}\right)\right]+T^{* *}\left[1-\exp \left(-\frac{t^{(k)}-t^{*}}{T^{* *}}\right)\right] \exp \left(-\frac{t^{*}-t_{0}}{T^{*}}\right), \\
\frac{\partial}{\partial \beta_{0}} \varphi^{* * *}\left(k^{* *} \leq k\right)=T^{*}\left[1-\exp \left(-\frac{t^{*}-t_{0}}{T^{*}}\right)\right]+T^{* *}\left[1-\exp \left(-\frac{t^{* *}-t^{*}}{T^{* *}}\right)\right] \exp \left(-\frac{t^{*}-t_{0}}{T^{*}}\right) \\
+T^{* * *}\left[1-\exp \left(-\frac{t^{(k)}-t^{* *}}{T^{* * *}}\right)\right] \exp \left(-\frac{t^{* *}-t^{*}}{T^{* *}}\right) \exp \left(-\frac{t^{*}-t_{0}}{T^{*}}\right)
\end{gathered}
$$

derivative with respect to $\beta^{*}$

$$
\begin{gathered}
\frac{\partial}{\partial \beta^{*}} \varphi^{*}\left(k<k^{*}\right)=\left(t^{(k)}-t_{0}\right)-T^{*}\left[1-\exp \left(-\frac{t^{(k)}-t_{0}}{T^{*}}\right)\right], \\
\frac{\partial}{\partial \beta^{*}} \varphi^{* *}\left(k^{*} \leq k<k^{* *}\right)=\left(t^{*}-t_{0}\right)-T^{*}\left[1-\exp \left(-\frac{\left.t^{*}-t_{0}\right)}{T^{*}}\right)\right] \\
+T^{* *}\left[1-\exp \left(-\frac{t^{(k)}-t^{*}}{T^{* *}}\right)\right]\left[1-\exp \left(-\frac{t^{*}-t_{0}}{T^{*}}\right)\right], \\
\frac{\partial}{\partial \beta^{*}} \varphi^{* * *}\left(k^{* *} \leq k\right)=\left(t^{*}-t_{0}\right)-T^{*}\left[1-\exp \left(-\frac{t^{*}-t_{0}}{T^{*}}\right)\right]+T^{* *}\left[1-\exp \left(-\frac{t^{* *}-t^{*}}{T^{* *}}\right)\right]\left[1-\exp \left(-\frac{t^{*}-t_{0}}{T^{*}}\right)\right] \\
+T^{* * *}\left[1-\exp \left(-\frac{t^{(k)}-t^{* *}}{T^{* * *}}\right)\right] \exp \left(-\frac{t^{* *}-t^{*}}{T^{* *}}\right)\left[1-\exp \left(-\frac{t^{*}-t_{0}}{T^{*}}\right)\right] ;
\end{gathered}
$$

derivative with respect to $\beta^{* *}$

$$
\begin{gathered}
\frac{\partial}{\partial \beta^{* *}} \varphi^{*}\left(k<k^{*}\right)=0, \\
\frac{\partial}{\partial \beta^{* *}} \varphi^{* *}\left(k^{*} \leq k<k^{* *}\right)=\left(t^{(k)}-t^{*}\right)-T^{* *}\left[1-\exp \left(-\frac{t^{(k)}-t^{*}}{T^{* *}}\right)\right],
\end{gathered}
$$




$$
\begin{gathered}
\frac{\partial}{\partial \beta^{* *}} \varphi^{* * *}\left(k^{* *} \leq k\right)=\left(t^{* *}-t^{*}\right)-T^{* *}\left[1-\exp \left(-\frac{t^{* *}-t^{*}}{T^{* *}}\right)\right] \\
+T^{* * *}\left[1-\exp \left(-\frac{t^{(k)}-t^{* *}}{T^{* * *}}\right)\right]\left[1-\exp \left(-\frac{t^{* *}-t^{*}}{T^{* *}}\right)\right] ;
\end{gathered}
$$

derivative with respect to $\beta^{* * *}$

$$
\begin{gathered}
\frac{\partial}{\partial \beta^{* * *}} \varphi^{*}\left(k<k^{*}\right)=0, \quad \frac{\partial}{\partial \beta^{* * *}} \varphi^{* *}\left(k^{*} \leq k<k^{* *}\right)=0, \\
\frac{\partial}{\partial \beta^{* * *}} \varphi^{* * *}\left(k^{* *} \leq k\right)=\left(t^{(k)}-t^{* *}\right)-T^{* * *}\left[1-\exp \left(-\frac{t^{(k)}-t^{* *}}{T^{* * *}}\right)\right] ;
\end{gathered}
$$

derivative with respect to $T^{*}$

$$
\begin{gathered}
\frac{\partial}{\partial T^{*}} \varphi^{*}\left(k<k^{*}\right)=\left(\beta_{0}-\beta^{*}\right)\left\{1-\left(1+\frac{t^{(k)}-t_{0}}{T^{*}}\right) \exp \left(-\frac{t^{(k)}-t_{0}}{T^{*}}\right)\right\}, \\
\frac{\partial}{\partial T^{*}} \varphi^{* *}\left(k^{*} \leq k<k^{* *}\right)=\left(\beta_{0}-\beta^{*}\right)\left[1-\left(1+\frac{t^{*}-t_{0}}{T^{*}}\right) \exp \left(-\frac{t^{*}-t_{0}}{T^{*}}\right)\right] \\
+\left(\beta_{0}-\beta^{*}\right) \frac{T^{* *}}{T^{*}}\left[1-\exp \left(-\frac{t^{(k)}-t^{*}}{T^{* *}}\right)\right] \frac{t^{*}-t_{0}}{T^{*}} \exp \left(-\frac{t^{*}-t_{0}}{T^{*}}\right), \\
\frac{\partial}{\partial T^{*}} \varphi^{* * *}\left(k^{* *} \leq k\right)=\left(\beta_{0}-\beta^{*}\right)\left[1-\left(1+\frac{t^{*}-t_{0}}{T^{*}}\right) \exp \left(-\frac{t^{*}-t_{0}}{T^{*}}\right)\right] \\
+\left(\beta_{0}-\beta^{*}\right) \frac{T^{* *}}{T^{*}}\left[1-\exp \left(-\frac{t^{* *}-t^{*}}{T^{* *}}\right)\right] \frac{t^{*}-t_{0}}{T^{*}} \exp \left(-\frac{t^{*}-t_{0}}{T^{*}}\right) \\
+\left(\beta_{0}-\beta^{*}\right) \frac{T^{* * *}}{T^{*}}\left[1-\exp \left(-\frac{t^{(k)}-t^{* *}}{T^{* * *}}\right)\right] \exp \left(-\frac{t^{* *}-t^{*}}{T^{* *}}\right) \frac{t^{*}-t_{0}}{T^{*}} \exp \left(-\frac{t^{*}-t_{0}}{T^{*}}\right) ;
\end{gathered}
$$

derivative with respect to $T^{* *}$

$$
\begin{gathered}
\frac{\partial}{\partial T^{* *}} \varphi^{*}\left(k<k^{*}\right)=0, \\
\frac{\partial}{\partial T^{* *}} \varphi^{* *}\left(k^{*} \leq k<k^{* *}\right)=\left[\beta\left(t^{*}\right)-\beta^{* *}\right]\left[1-\left(1+\frac{t^{(k)}-t_{0}}{T^{* *}}\right) \exp \left(-\frac{t^{(k)}-t_{0}}{T^{* *}}\right)\right], \\
\frac{\partial}{\partial T^{* *}} \varphi^{* * *}\left(k^{* *} \leq k\right)=\left[\beta\left(t^{*}\right)-\beta^{* *}\right]\left[1-\left(1+\frac{t^{* *}-t^{*}}{T^{* *}}\right) \exp \left(-\frac{t^{* *}-t^{*}}{T^{* *}}\right)\right] \\
+\left[\beta\left(t^{*}\right)-\beta^{* *}\right] \frac{t^{* *}-t^{*}}{T^{* *}} \frac{T^{* * *}}{T^{* *}}\left[1-\exp \left(-\frac{t^{(k)}-t^{* *}}{T^{* * *}}\right)\right] \exp \left(-\frac{t^{* *}-t^{*}}{T^{* *}}\right)
\end{gathered}
$$


derivative with respect to $T^{* * *}$

$$
\begin{gathered}
\frac{\partial}{\partial T^{* * *}} \varphi^{*}\left(k<k^{*}\right)=0, \quad \frac{\partial}{\partial T^{* * *}} \varphi^{* *}\left(k^{*} \leq k<k^{* *}\right)=0 \\
\frac{\partial}{\partial T^{* * *}} \varphi^{* * *}\left(k^{* *} \leq k\right)=\left[\beta\left(t^{* *}\right)-\beta^{* * *}\right]\left[1-\left(1+\frac{t^{(k)}-t^{* *}}{T^{* * *}}\right) \exp \left(-\frac{t^{(k)}-t^{* *}}{T^{* * *}}\right)\right] ;
\end{gathered}
$$

derivative with respect to $\ln N_{0}$

$$
\frac{\partial}{\partial \ln N_{0}} \varphi^{*}\left(k<k^{*}\right)=\frac{\partial}{\partial \ln N_{0}} \varphi^{* *}\left(k^{*} \leq k<k^{* *}\right)=\frac{\partial}{\partial \ln N_{0}} \varphi^{* * *}\left(k^{* *} \leq k\right)=1 .
$$

